\title{
Selective versus routine lymphadenectomy in the treatment of liver metastasis from colorectal cancer: a retrospective cohort study
}

\author{
Daniel Pindak ${ }^{1,2^{*}}$ D, Jana Pavlendova ${ }^{1,2}$, Miroslav Tomas ${ }^{1,2}$, Jozef Dolnik ${ }^{2}$, Robert Duchon ${ }^{2}$ and Juraj Pechan ${ }^{1,2}$
}

\begin{abstract}
Background: Limited data are available on the importance of routine lymphadenectomy of the hepatoduodenal ligament in the treatment of liver metastasis from colorectal cancer in the literature.

Methods: A single center retrospective cohort study was conducted to evaluate morbidity and long-term survival in patients who had undergone selective versus routine lymphadenectomy during surgery for colorectal liver metastasis. From January 2006 to December 2009, eighty-one patients undergoing radical resection due to liver metastasis from colorectal cancer were included. The combination of two surgical teams with different approaches to hepatoduodenal ligament lymphadenectomy at our institution allowed us to select two cohorts of patients undergoing selective or routine lymphadenectomy.

Results: No significant differences between the cohorts were found in age, American Society of Anesthesiology score or Fong's prognostic criteria. Patients with pN+ disease had significantly inferior survival compared to patients with pNO disease (hazard ratio $[\mathrm{HR}]=6.33,95 \% \mathrm{Cl} 2.16-18.57, p=0.0001$ ). No significant difference in postoperative morbidity was observed in the group undergoing routine opposed to selective lymphadenectomy (13.63\% vs. $8.69 \%, p=0.36)$. There was no difference in long-term survival between the groups $(H R=0.90,95 \% \mathrm{Cl} 0.52-1$. $58, p=0.70)$. There were also no significant differences in the subgroup of patients with pNO stage ( $H R=1.17$, $95 \% \mathrm{Cl} 0.6-2.11, p=0.60)$.

Conclusions: These data suggest that there is no survival benefit from the use of routine lymphadenectomy during surgery for colorectal liver metastasis, but these data should be confirmed in a prospective randomized study.
\end{abstract}

Keywords: Colorectal, Liver, Metastasis, Lymphadenectomy, Survival

\section{Background}

The liver is the most common site of metastatic spread after curative treatment of colorectal cancer (CRC), and approximately $50 \%$ of all patients with CRC will develop liver metastases [1]. Liver resection represents the best treatment option, with a long-term survival rate of $30 \%$. Resectability is recommended only in approximately $20 \%$ of patients, mainly due to the presence of extrahepatic disease [2]. The infiltration of lymph nodes (LN) in

\footnotetext{
* Correspondence: pindakd@zoznam.sk; daniel.pindak@nou.sk ${ }^{1}$ Department of Surgical Oncology, Slovak Medical University, Bratislava, Slovakia

${ }^{2}$ National Cancer Institute, Klenova 1, 83310 Bratislava 3, Slovakia
}

the hepatic pedicle and the coeliac region is considered an extrahepatic disease, and many authors still consider its involvement to be a contraindication for liver resection, especially if they are in the coeliac region. Limited data are available on the importance of routine lymphadenectomy of the hepatoduodenal ligament in the literature. Moreover, its survival benefit for both prophylactic and therapeutic indications is not clear [2]. To date, there have been no prospective randomized studies published on this topic. The combination of two different surgical teams with different approaches to lymphadenectomy during liver resection in CRC, at the National Cancer Institute Bratislava Slovakia (NCI), gave us the 
opportunity to conduct a study with the aim of evaluating the role of routine lymphadenectomy during liver resection in patients with CRC.

\section{Methods}

This retrospective cohort study was conducted to determine the influence of routine lymphadenectomy of the hepatoduodenal ligament on postoperative morbidity and long-term survival in patients with clinically negative nodal status in the hepatoduodenal ligament according to preoperative computed tomography $(\mathrm{CT})$ scans.

\section{Patient selection}

From January 2006 to December 2009, eighty-one patients underwent liver resection due to CRC metastases at the National Cancer Institute Bratislava. Patients were divided into two cohorts: patients with or without a routine lymphadenectomy (Fig. 1). This was possible due to the existence of two different hepatobiliary surgical teams working during that time period in the NCI with different approaches to lymphadenectomy. At that time, the presence of nodal involvement in the hepatic pedicle and coeliac region was considered a contraindication for hepatic resection, so all patients who underwent liver resection had clinically negative nodes. At that time, no patient received neoadjuvant or "conversion" chemotherapy before hepatic resection; thus, its influence is of no concern for survival analysis. Administration of adjuvant chemotherapy was considered individually, on the basis of an overall assessment of the below-mentioned prognostic factors. The most commonly used regimen was fluoropyrimidine plus oxaliplatin. Unfortunately different institutions in Slovakia delivered subsequent oncological treatment, so we did not have data about adjuvant chemotherapy available in all patients.

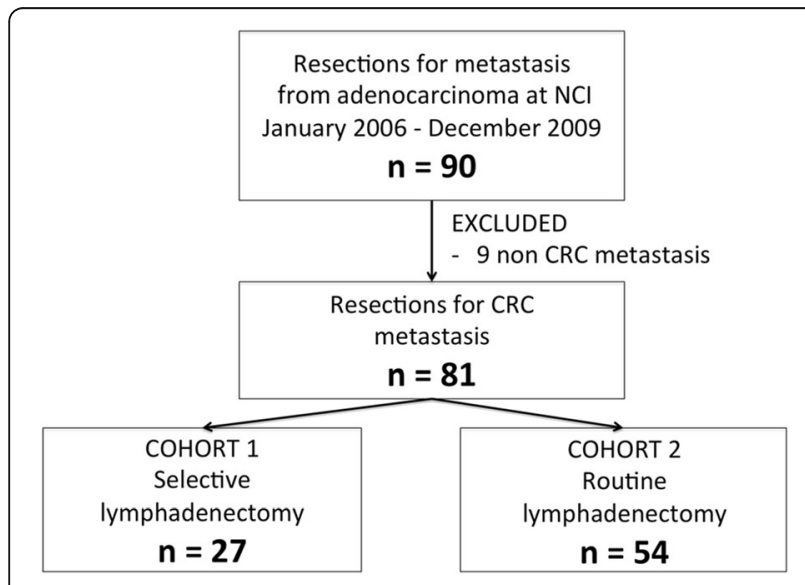

Fig. 1 Patients' selection

\section{Data collection}

From the patient's medical records and the National Center of Medical Information and Population Register, we constructed specific case report forms with clinical features and survival information. These features included age, gender, American Society of Anesthesiologists (ASA) score, number and size of liver metastases, disease-free interval (from the treatment of the CRC primary tumor), level of preoperative carcinoembryonic antigen (CEA), positivity of pathological nodal staging $(\mathrm{pN}+)$ of the primary tumor, definitive histology finding (only hematoxylin and eosin staining was used for the evaluation of nodal status), presence of postoperative complications and their classification according to the Clavien Dindo system [3], 30-day postoperative mortality and overall survival.

\section{Surgical technic}

In cohort 1, no standard lymphadenectomy was performed; nodal sampling (selective lymphadenectomy) was performed only if there was a suspicion for nodal involvement according to macroscopic assessment or intraoperative palpation. In cohort 2, routine lymphadenectomy that included nodes in hepatoduodenal ligament and along the common hepatic artery, coeliac trunk and retropancreatic region was performed.

\section{Statistical analysis}

Statistical relationships were evaluated by different tests depending on the data characteristics (KolmogorovSmirnov, $t$ test, Fisher-Freeman-Halton test), and Kaplan-Meier's method and Log-rank test were used to evaluate differences between the groups in terms of survival analysis. All of the statistical hypotheses were tested with a significance level of alpha $=0.05$, and the data cut-off for survival analysis was 31 May 2016. The study was approved by the institutional review board of the NCI Bratislava, Slovakia, and a waiver of consent was granted.

\section{Results}

\section{Cohort characteristics}

There were 55 men (66.7\%) and 26 women (33.3\%) included in the study. The median age was similar in both cohorts: 57 (42-88) versus 61 (38-82) years old, respectively $(p=0.63)$. The ASA score was also not different between the groups $(p=0.42)$. The most important and well-accepted prognostic criteria (metastasis size, number of metastases, preoperative CEA level, disease free interval from the treatment of the primary CRC and the nodal status of primary (RC), as counted both separately and by Fong's clinical score [4], were equal and exhibited no significant differences $(p=0.08)$. All of the data are shown in Table 1. The cohorts were well 
Table 1 Cohort comparisons with no significant differences in any prognostic factors

\begin{tabular}{|c|c|c|c|}
\hline & Cohort 1 & Cohort 2 & \multirow[t]{3}{*}{$p$ value } \\
\hline & $\begin{array}{l}\text { Selective } \\
\text { lymphadenectomy }\end{array}$ & $\begin{array}{l}\text { Routine } \\
\text { lymphadenectomy }\end{array}$ & \\
\hline & $n=27$ & $n=54$ & \\
\hline Age median (min/max) & $57(42-88)$ & $61(38-82)$ & $0.63^{\mathrm{a}}$ \\
\hline ASA score $(1 / 2 / 3 / 4) \%$ & $5 / 68 / 27 / 0$ & $18 / 68 / 14 / 0$ & $0.42^{\mathrm{a}}$ \\
\hline Fong's score $(0-2) /(3-5) \%$ & $96.97 / 3.03$ & $83.33 / 16.67$ & $0.08^{b}$ \\
\hline MTS size $(\mathrm{mm})$ median (min/max) & $21(10-40)$ & $20(8-130)$ & $0.12^{a}$ \\
\hline MTS number median (min/max) & $1(1-8)$ & $2(1-7)$ & $0.25^{\mathrm{a}}$ \\
\hline CEA level $\mu \mathrm{g} / \mathrm{l}$ median (min/max) & $5.7(0.3-850)$ & $6.62(0.4-850)$ & $0.97^{\mathrm{a}}$ \\
\hline $\mathrm{pN}+$ primary $\%$ & 41.67 & 29.09 & $0.29^{a}$ \\
\hline disease free $<12$ month $\%$ & 70.37 & 74.07 & $0.14^{\mathrm{a}}$ \\
\hline
\end{tabular}

balanced regarding known prognostic factors. The mean number of lymph nodes harvested in patients with routine lymphadenectomy was 4.6 (2-13) per patient. Overall, only one lymph node was sampled in 5 patients in the cohort with selective lymphadenectomy.

\section{Postoperative morbidity}

We found a higher rate of postoperative complications in the group of patients undergoing routine lymphadenectomy (13.63\%) compared to the group of patients with selective lymphadenectomy (8.69\%), but this difference did not reach statistical significance $(p=0.36)$.

The severity of complications evaluated by Clavien Dindo classification system also showed no significant differences $(p=0.61)$.

\section{Survival}

The median length of follow up was 61.67 (0.33-122.93) months.

There were 6 patients with positive nodes in cohort 2 (routine lymphadenectomy) and 1 patient with positive nodes in cohort 1 (selective lymphadenectomy), for a prevalence of $11.11 \%$ and $3.7 \%$ of histological nodal positivity, respectively. Using the Kaplan-Meier method and Log-rank test, we found a significant difference in survival between the patients with positive and negative nodes (median overall survival (mOS) 29.6 vs. 66.97 months, respectively, hazard ratio (HR) $6.3395 \%$ CI 2.16-18.57, $p=0.0001$ ) (Fig. 2).

There were no significant differences in long-term survival between cohorts 1 and 2 (median survival 61.62 vs. 60.16 months, respectively, HR 0.90 95\% CI 0.52-1.58, $p=0.7$ ) (Fig. 3). The 3- and 5-year survival rates were 59.25 and $51.85 \%$ vs. 66.67 and $48.15 \%$ in cohorts 1 and 2 , respectively.

To evaluate the prophylactic potential of lymphadenectomy, we selected patients with pN0 nodal status

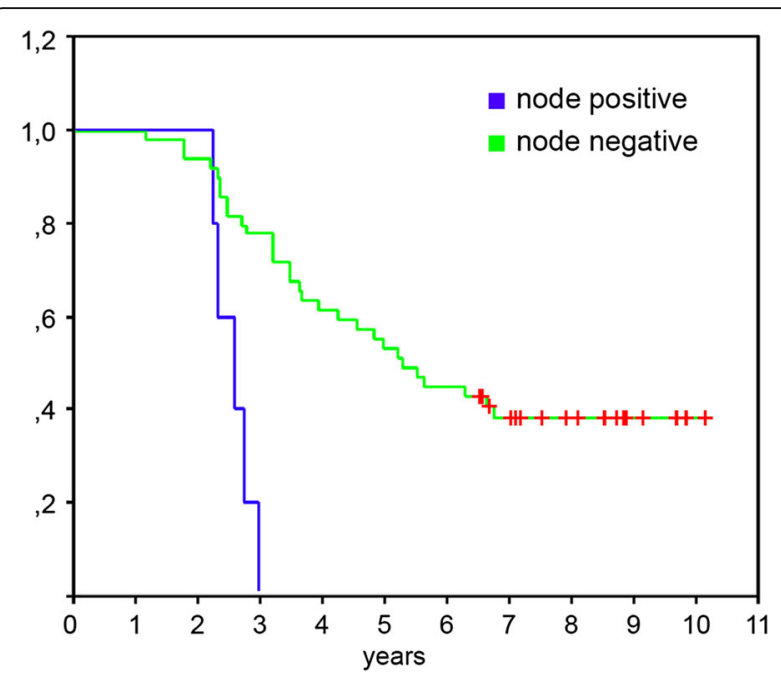

Fig. 2 Survival according to histological nodal status. Kaplan-Meier curves showing the difference in overall survival in patients with positive lymph nodes in hepatic pedicle $(n=7)$ compared to patients with negative nodes $(n=74)$. The green line indicates patients with negative nodes, the blue line patients with positive nodes. The vertical lines on the curve mark censored data. The difference is statistically significant HR $6.3395 \%$ Cl 2.16-18.57, $p=0.0001$

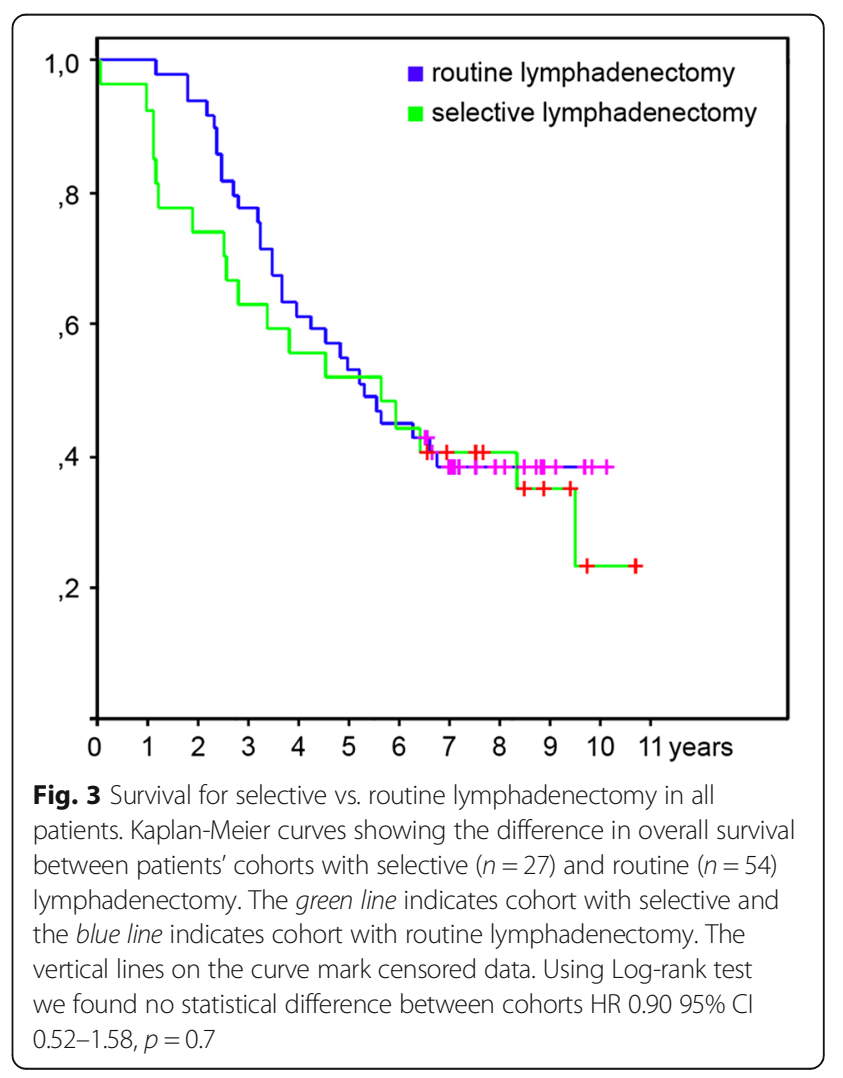


after definitive histology and found no significant difference in survival (HR 1.17 95\% CI $0.6-2.11, p=0.6$ ) (Fig. 4).

\section{Discussion}

The indication for lymphadenectomy of the hepatoduodenal ligament during liver resection for CRC metastasis remains controversial. It is still unclear whether the lymph node dissection imparts a survival advantage or merely allows a more complete staging of the disease [2]. The metastatic involvement of nodes is considered one of the worst prognostic factors $[5,6]$ and was once a contraindication for liver resection because the 5 year survival ranged between 0 and $4.3 \%[7,8]$. The location of the involved nodes also played an important role, as stated by Adam et al: for those who had metastases in the hepatic pedicle and underwent hepatectomy, the 5 year survival was better (25\%) than that observed in patients with positive nodes in the coeliac and paraaortic regions (0\%) [9]. In our study, we performed en bloc lymphadenectomy, so the subclassification of nodes to different stations was not possible, but none of our node-positive patients survived for 3 years, despite the use of adjuvant chemotherapy. We can therefore confirm that nodal positivity is a negative prognostic factor: node-positive patients exhibited a median survival of 29.6 months vs. 66.97 for node-negative patients.

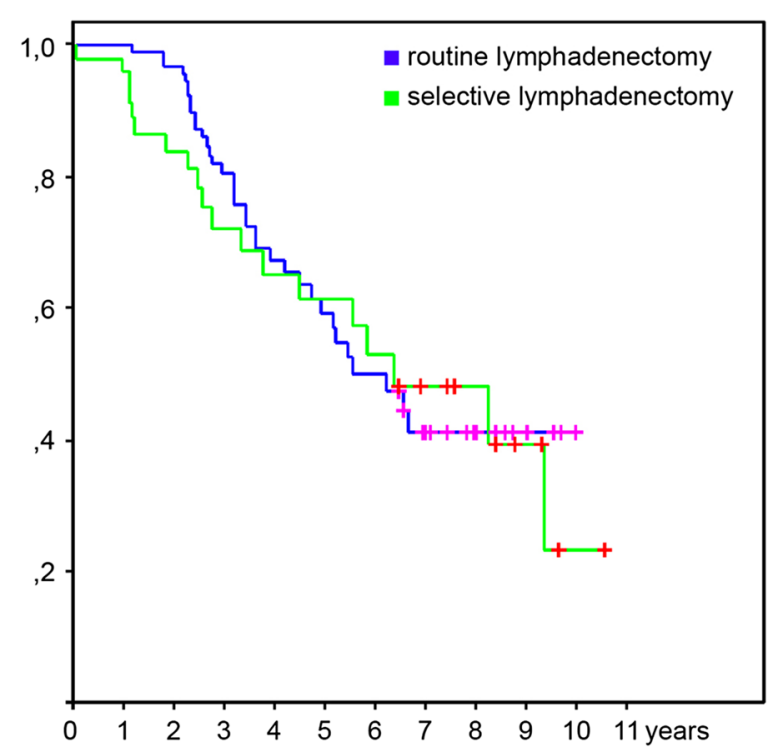

Fig. 4 Survival for selective vs. routine lymphadenectomy in pNO patients. Kaplan-Meier curves showing the difference in overall survival in the subgroup of patients with confirmed pNO stage. The green line indicates cohort with selective and the blue line indicates cohort with routine lymphadenectomy. The vertical lines on the curve mark censored data. Using Log-rank test we found no significant difference in survival HR $1.1795 \% \mathrm{Cl} 0.6-2.11, p=0.6$ between patients' cohorts with selective $(n=26)$ and routine $(n=48)$ lymphadenectomy
The overall prevalence of positive lymph nodes was reported to be $8.4 \%$, ranging between 5.4 and $27.9 \%$ after routine lymphadenectomy and between 4.0 and $22.5 \%$ after selective lymphadenectomy. Koti et al. reported that the prevalence was not correlated to the extent or approach (routine/selective) of lymphadenectomy [10]. In our study, the prevalence was higher in the group of patients undergoing routine lymphadenectomy compared to selective lymphadenectomy $(11.11 \%$ vs. $3.7 \%)$, which can be explained by patient selection for those with $\mathrm{cNO}$ stage. During the study period, the clinical positivity of extrahepatic nodes was considered a contraindication for liver resection.

Regardless of the extent of lymphadenectomy that was performed, we found equal survival in patients with and without routine lymphadenectomy (median survival 61.62 vs. 60.16 months, respectively, HR $0.9095 \%$ CI $0.52-1.58, p=0.7)$.

To evaluate the "prophylactic" potential of lymphadenectomy, we excluded all patients with proven positive nodes. We assumed, that routine lymphadenectomy in patients with pNO nodal status could improve survival through the eliminating potential site of future recurrence, as in the cohort with selective lymphadenectomy, patients were expected to have residual nodal disease. We also found no survival advantage of routine lymphadenectomy over no lymphadenectomy (HR 1.17 95\% CI 0.6-2.11).

Although the routine lymphadenectomy of the hepatoduodenal ligament in the treatment of liver metastasis from CRC is, according to our results, a safe procedure with acceptable morbidity that is not higher than after liver resection only ( $p=0.36$ ), we found no survival benefit from its use. Therefore, we suggest considering routine lymphadenectomy during liver resection individually until evidence from high-quality studies supports its use.

Our results do not support the use of routine lymphadenectomy; therefore, we suggest to not perform it as a standard procedure, although its morbidity is the same as after liver resection only $(p=0.36)$. The quality of data in the literature is unclear, and the conclusions are controversial; thus, we suggest that patients be enrolled in prospective clinical studies, which we are currently performing.

\section{Conclusion}

Although the routine lymphadenectomy of the hepatoduodenal ligament in the treatment of liver metastasis from CRC is, according to our results, a safe procedure with acceptable morbidity, we found no survival benefit from its use. Therefore, we suggest considering routine lymphadenectomy during liver resection individually until evidence from high-quality studies supports its use. We confirmed the presence of nodal metastasis as a negative prognostic marker with zero percent 3-year survival. 


\section{Abbreviations}

ASA: American Society of Anesthesiologists; CEA: Carcinoembryonic antigen; CRC: Colorectal cancer; CT: Computed tomography; HR: Hazard ratio; LN: Lymph nodes; NCl: National Cancer Institute Bratislava Slovakia; $\mathrm{pN}+$ : positivity of pathological nodal staging; vs.: versus

\section{Acknowledgements}

Special thanks to Dr. Michal Mego from $\mathrm{NCl}$ for reviewing important intellectual content and language corrections.

\section{Funding}

Not applicable.

\section{Availability of data and materials}

All data generated or analyzed during this study are included in this published article.

\section{Authors' contributions}

Authors contributed to the conception and design (DP, JP), to the writing of the manuscript (DP), and the acquisition of data, statistical analysis and interpretation of data (JP, DP, MT). All authors read and approved the final manuscript.

\section{Competing interests}

The authors declare that they have no competing interests.

\section{Consent for publication}

Not applicable.

\section{Ethics approval and consent to participate}

The study was approved by the institutional review board and ethics committee of the National Cancer Institute Bratislava, Slovakia and a waiver of consent was granted.

\section{Publisher's Note}

Springer Nature remains neutral with regard to jurisdictional claims in published maps and institutional affiliations.

Received: 6 October 2016 Accepted: 31 March 2017

Published online: 04 April 2017

\section{References}

1. Gurusamy KS, Imber C, Davidson BR. Management of the hepatic lymph nodes during resection of liver metastases from colorectal cancer: a systematic review. HPB Surg. 2008; doi:10.1155/2008/684150

2. Bradatsch A, Kornprat P, Bacher $H$, Cerwenka $H$, Haybaeck J, Mischinger $H J$. The Value of Lymph Node Dissection in the Surgery of Colorectal Cancer Liver Metastases. Anticancer Res. 2016;36:2993-7.

3. Dindo D, Demartines N, Clavien PA. Classification of surgical complications: a new proposal with evaluation in a cohort of 6336 patients and results of a survey. Ann Surg. 2004;240(2):205-13.

4. Fong Y, Fortner J, Sun RL, Brennan MF, Blumgart LH. Clinical score for predicting recurrence after hepatic resection for metastatic colorectal cancer: analysis of 1001 consecutive cases. Ann Surg. 1999;230(3):309-18.

5. Rodgers MS, McCall JL. Surgery for colorectal liver metastases with lymph node involvement: a systematic review. Br J Surg. 2000; doi:10.1046/j.13652168.2000.01580.x

6. Poston GJ, Adam R, Alberts S, Curley S, Figueras J, Haller D, et al. OncoSurge: a strategy for improving resectability with curative intent in metastatic colorectal cancer. J Clin Oncol. 2005; doi:10.1200/JCO.2005.08.722

7. Jaeck D, Nakano H, Bachellier P, Inoue K, Weber JC, Oussoultzoglou E, et al. Significance of hepatic pedicle lymph node involvement in patients with colorectal liver metastases: a prospective study. Ann Surg Oncol. 2002;9(5):430-8.

8. Laurent C, Cunha AS, Rullier E, Smith D, Rullier A, Saric J. Impact of microscopic hepatic lymph node involvement on survival after resection of colorectal liver metastasis. J Am Coll Surg. 2004; doi: 10.1016/j.jamcollsurg. 2004.01.017

9. Adam R, de Haas RJ, Wicherts DA, Aloia TA, Delvart V, Azoulay D, et al. Is hepatic resection justified after chemotherapy in patients with colorectal liver metastases and lymph node involvement? J clin Oncol. 2008, doi:10.1200/JCO.2007.15.7297

10. Koti RS, Simillis C, Gurusamy KS. Jacovides M, Davidson BR. Management of the Hepatic Lymph Nodes During Resection of Liver Metastases from Colorectal Cancer: A Systematic Review. Curr Colorectal Cancer Rep. 2013; doi: 10.1007/s11888-013-0165-6

\section{Submit your next manuscript to BioMed Central and we will help you at every step:}

- We accept pre-submission inquiries

- Our selector tool helps you to find the most relevant journal

- We provide round the clock customer support

- Convenient online submission

- Thorough peer review

- Inclusion in PubMed and all major indexing services

- Maximum visibility for your research

Submit your manuscript at www.biomedcentral.com/submit
) Biomed Central 